ISSN 0819-2642

ISBN 0734026110

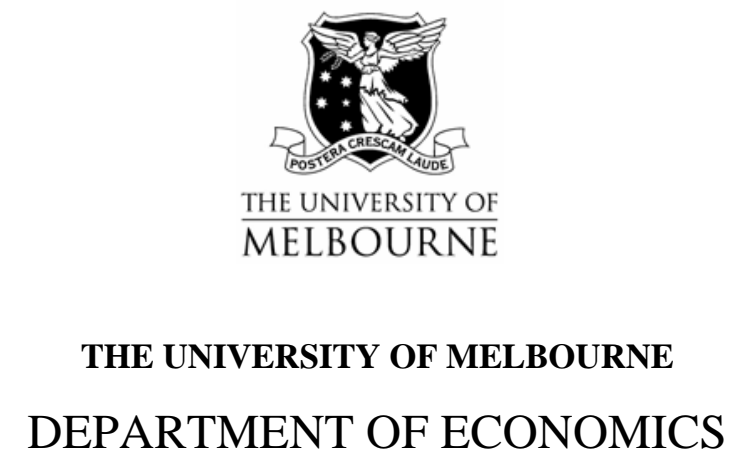

RESEARCH PAPER NUMBER 954

NOVEMBER 2005

\title{
ALTERNATIVE FORMS FOR RESTRICTED REGRESSIONS
}

by

Joe Hirschberg

\&

Jenny Lye

\&

Daniel J. Slottje

Department of Economics

The University of Melbourne Melbourne Victoria 3010

Australia. 


\title{
Alternative Forms for Restricted Regressions ${ }^{1}$
}

\author{
by \\ Joe Hirschberg and Jenny Lye \\ Department of Economics \\ University of Melbourne \\ Parkville, Victoria \\ Australia \\ Daniel J. Slottje \\ Department of Economics \\ Southern Methodist University \\ Dallas, Texas 75275-0496
}

April, 2005

Key words

Linear Restrictions, Reparameterization, Polynomial lags, Spline lags

Classification

C51, D12, C63

\begin{abstract}
The estimation of regression models subject to linear restrictions, is a widely applied technique, however, aside from simple examples, the equivalence between the linear restricted case to the reparameterization or substitution case is rarely employed. We believe this is due to the lack of a general transformation method for changing from the definition of restrictions in terms of the unrestricted parameters to the equivalent reparameterized model and conversely, from the reparameterized model to the equivalent linear restrictions for the unrestricted model. In many cases the reparameterization method is computationally more efficient especially when estimation involves an iterative method. But the linear restriction case allows a simple method for adding and removal of restrictions.

In this paper we derive a general relationship that allows the conversion between the two forms of the restricted models. Examples involving systems of demand equations, polynomial lagged equations, and splines are given in which the transformation from one form to the other are demonstrated as well as the combination of both forms of restrictions. In addition, we demonstrate how an alternative Wald test of the restrictions can be constructed using an augmented version of the reparameterized model.
\end{abstract}

\footnotetext{
${ }^{1}$ We wish to acknowledge Thomas B. Fomby for helpful suggestions and comments on this paper, the usual caveat holds.
} 


\section{Introduction}

Regression models that are the subject of exact linear restrictions is a well defined topic in most econometrics textbooks (such as Griffiths et al 1993, Greene 2003, Johnston and DiNardo 1997, and Ruud 2000). With the notable exception of Ruud, the exposition in these texts has changed little since Tintner's (1952) text. However, in many of these treatments the restricted and unrestricted parameter vectors are given the same names (i.e. $\beta$ ), although the estimates are defined differently (i.e. $\hat{\beta}$ for the unrestricted and $\hat{\beta}^{*}$ or $\hat{\beta}_{r}$ for the restricted estimates) thus confusing both the implications of the restrictions on the model and on estimation.

In this paper we present a formulation to this problem that emphasizes an aspect of restricted regressions that is often less obvious, namely that the model specified has been changed by the restriction. We focus on the implications for the model specification of the linear restrictions and sets of linear restrictions and derive an automatic procedure for translating a problem from the linear restrictions of the unrestricted parameter vector form to the reparameterization of the unrestricted parameter form and back. Finally, we provide examples of how this transformation can be applied to single and multiple restrictions and show how the reparameterized or substitution form can be used to test restrictions.

The correspondence between the linear restrictions form and a reparameterized (also referred to as substitution) form has been noted in numerous contributions to the literature. Mantell (1973) showed that it is always possible to reconfigure a set of linear restrictions on the vector of regression coefficients in such a way as to form an equivalent regression defined in terms of a new vector of unrestricted parameters and more recent econometrics texts report this result. Fomby et. al. (1984), Greene (2003), Davidson and MacKinnon (1993) and Griffiths et al (1993) all present the case of linear restrictions applied to the unrestricted parameters. In a more recent text Ruud (2000) devotes an entire chapter (chapter 4) to the case of restricted least squares by proposing that the restrictions are in the form of a reparameterization while only 
noting in passing equivalence to the traditional linear function of the unrestricted parameters. However, none of these treatments present a general method for the derivation of the alternative reparameterized model from the linear restrictions and back again. The only proposal for the conversion involves an unspecified method to decompose the restrictions matrix. The applications in which this interrelationship between the two forms have been used employ ad hoc methods for conversion which apply only to the particular cases at hand (i.e. Huang and Haidacher 1983 and Fomby et al 1984 pp 377-378). Given the lack of a general method that uses standard software to make this translation, it is not surprising that the reparameterization method is rarely used unless it is required for estimation. Additionally, it is rare to find cases where the reparameterized problem has been translated to the restrictions of unrestricted parameters form.

The contribution of this paper is to derive the general form of the correspondence between these two ways of representing a restricted regression model. The relationship defined here allows the transformation of restrictions defined in either way to be defined by the alternative. In addition, this correspondence is one that can be easily automated because it requires only simple matrix manipulations and the singular value decomposition of a symmetric matrix.

This paper proceeds as follows. First we define the two approaches as they have appeared in the literature and present the process for transformation between the forms. Next we provide a series of examples to demonstrate how these transformations may be used. In particular, we focus on the case where both forms of restrictions may be present at the same time. The last section of this paper demonstrates how the reparameterized solution can be used in the construction of a test of the restrictions which is equivalent to the tests performed in terms of the unrestricted parameters. The proofs of these correspondences are given in the appendices. 


\section{The Two Forms of Restrictions.}

A regression model subject to a set of linear restrictions is written in the form

$$
\mathbf{Y}=\mathbf{X}(\beta)+\varepsilon, \text { s.t. } \mathbf{R} \beta=\mathbf{r},
$$

where $\mathbf{Y}_{T \times 1}$ is the vector of the observations on the dependent variable, $\mathbf{X}(\cdot)$ is a vector of regression functions of length $T$ and $\beta_{k \times 1}$ is the set of restricted parameters, $\mathbf{R}_{m \times k}$ is the matrix of $m$ linear combinations of the restricted parameter set, $\mathbf{r}_{m \times 1}$ is the vector of constraints to which we equate the linear combinations, $m<k$ and the assumptions concerning the error $(\varepsilon)$ will depend on the case at hand. This form of the problem will be referred to as the "linear function of unrestricted parameters" form of the restrictions or the LFUP form. As noted above, numerous econometrics texts treat this as the only formulation of the restricted regression model. Simple econometric examples of this sort of restrictions would be the imposition of constant returns to scale in a Cobb-Douglas production function and the homogeneity of degree zero in the prices of a $\log \log$ demand equation.

The alternative method for defining linear restrictions is the case when $\beta$ can be written as a linear function of a set of $j(j=k-m)$ other parameters $(\gamma)$ and a $k \times 1$ vector of constants $(\mathbf{d})$ as

$$
\beta=\mathbf{A} \gamma+\mathbf{d}
$$

This form of linear restrictions will be referred to as ROP for "reparameterized in other parameters" form of the restrictions and the regression can be written as

$$
\mathbf{Y}=\mathbf{X}(\beta)+\boldsymbol{\varepsilon}, \text { s.t. } \beta=\mathbf{A} \gamma+\mathbf{d}
$$

which, by substitution, is equivalent to the reparameterized regression written as

$$
\mathbf{Y}=\mathbf{Z}(\gamma)+\boldsymbol{\varepsilon}
$$

where $\mathbf{Z}(\gamma)=\mathbf{X}(\mathbf{A} \gamma+\mathbf{d})$. This form of a restricted regression model is also found in the econometrics literature though much less often (e.g. Ruud 2000 pp. 77 - 84).

An advantage of the reparameterization method is the ability to derive a linear equation such as (2) that relates the $k$ by 1 vector $\beta$ to the transformed set of $j$ parameters $(\gamma)$. The explicit 
definition of $\beta$ as a function of $\gamma$ serves to maintain the separation of the parameters subject to restrictions $(\beta)$ from those that are "free" $(\gamma)$, or more precisely those that are not subjected to restrictions.

It is the relationship between the LFUP and the ROP which we are concerned with in this paper. The remainder of this section will examine the conversions from the LFUP form to the ROP form and from the ROP form to the LFUP form and the computational aspects of this conversion.

\subsection{LFUP to $\operatorname{ROP}(\mathrm{R} \beta=\mathrm{r}) \rightarrow(\beta=\mathbf{A} \gamma+\mathbf{d})$}

A is defined as the $k$ by $j$ matrix of $j$ eigenvectors corresponding to the zero valued eigenvalues of $\mathbf{R}^{\prime} \mathbf{R}$ and $\mathbf{d}$ is defined as $\mathbf{R}^{+} \mathbf{r}$. When the rank of $\mathbf{R}$ is equal to $m$ (thus $k=m+j$ ), an appropriate Moore-Penrose g-inverse $\left(\mathbf{R}^{+}\right)$is given by

$$
\mathbf{R}^{+}=\mathbf{R}^{\prime}\left(\mathbf{R} \mathbf{R}^{\prime}\right)^{-1}
$$

The proof of this relationship is given in Appendix A.

The estimated restricted parameters $(\tilde{\beta})$, can be found by using (2) and the estimates for $\gamma$ as estimated from (4) and the corresponding covariance is given as

$$
\operatorname{cov}(\tilde{\beta})=\mathbf{A}(\operatorname{cov}(\hat{\gamma})) \mathbf{A}^{\prime}
$$

Note that the reparameterized regression (4) is written with the same error, $\varepsilon$, as the original problem. Consequently, if the regression model is linear (i.e. $\mathbf{X}(\beta)=\mathbf{X} \beta)$ then the covariance matrix of the error is estimated as $\tilde{\Sigma}$, the equivalent solution to the GLS problem is;

$$
\tilde{\gamma}=\left(\mathbf{Z}^{\prime} \tilde{\Sigma}^{-1} \mathbf{Z}\right)^{-1} \mathbf{Z}^{\prime} \tilde{\Sigma}^{-1} \mathbf{G}
$$

where $\mathbf{Z}=\mathbf{X A}$ and $\mathbf{G}=\mathbf{Y}$ - Xd. In this case, the estimates of $\beta$ can then be defined via (2) with the GLS estimate of $\gamma$ and the covariance of $\beta$ can be defined in a form equivalent to (6) using the GLS estimated covariance for $\gamma$.

\subsection{ROP to LFUP $(\beta=\mathbf{A} \gamma+\mathbf{d} \rightarrow \mathrm{R} \beta=\mathrm{r})$}


In Appendix B we show that $\beta=\mathbf{A} \gamma+\mathbf{d}$ is equivalent to a set of $m(m=k-j)$ linear restrictions in the parameters in the form $\mathbf{R} \beta=\mathbf{r}$, where $\mathbf{R}$ is defined as an $m$ by $k$ matrix equal to the transpose of the eigenvectors of $\mathbf{A A}^{\prime}$ that correspond to the $m$ zero valued eigenvalues of $\mathbf{A A}^{\prime}$ and $\mathbf{r}=\mathbf{R d}$.

\subsection{Computational Aspects of the Restricted Parameter Estimates and The Conversion} of Restricted Forms.

A major consideration for the use of reparameterization is in the process of estimation. For example, most widely used statistical software packages do not allow the imposition of linear restrictions in the LFUP form when using logit, probit, ARIMA or other maximum likelihood or robust estimation methods, the reparameterized version may be the only practical way to impose restrictions on these model's estimates. Also the use of Bayesian methods for the construction of the posterior distribution of a parameter vector is simplified if the parameters are unconstrained, thus distributions of estimates of $\gamma$ are easier to construct than those for estimates of $\beta$. For example, Griffiths et al. (2000 p113) show how a seemingly unrelated regression (SUR) model with restrictions of the LFUP form can be re-formulated as an unrestricted SUR model which is a convenient form to use the Markov Chain Monte Carlo simulation for the empirical implementation of the Bayesian approach.

The traditional generalized least squares solution to the restricted linear equation problem specified in (1) when $\mathbf{X}(\beta)=\mathbf{X} \beta$ and $\Sigma$ is estimated by $\tilde{\Sigma}$ would be given as:

$$
\begin{aligned}
\hat{\beta}= & \left(\mathbf{X}^{\prime} \tilde{\Sigma}^{-1} \mathbf{X}\right)^{-1} \mathbf{X}^{\prime} \tilde{\Sigma}^{-1} \mathbf{Y}+ \\
& \left(\mathbf{X}^{\prime} \tilde{\Sigma}^{-1} \mathbf{X}\right)^{-1} \mathbf{R}\left(\mathbf{R}\left(\mathbf{X}^{\prime} \tilde{\Sigma}^{-1} \mathbf{X}\right)^{-1} \mathbf{R}^{\prime}\right)^{-1}\left(\mathbf{r}-\mathbf{R}\left(\mathbf{X}^{\prime} \tilde{\Sigma}^{-1} \mathbf{X}\right)^{-1} \mathbf{X}^{\prime} \tilde{\Sigma}^{-1} \mathbf{Y}\right)
\end{aligned}
$$

which implies that $\mathbf{Q}$ (where $\mathbf{Q}=\left(\mathbf{X}^{\prime} \tilde{\Sigma}^{-1} \mathbf{X}\right)^{-1}$ ) is non-singular. When $\Sigma=$ I this simplifies to:

$$
\hat{\beta}=\left(\mathbf{X}^{\prime} \mathbf{X}\right)^{-1} \mathbf{X}^{\prime} \mathbf{Y}+\left(\mathbf{X}^{\prime} \mathbf{X}\right)^{-1} \mathbf{R}\left(\mathbf{R}\left(\mathbf{X}^{\prime} \mathbf{X}\right)^{-1} \mathbf{R}^{\prime}\right)^{-1}\left(\mathbf{r}-\mathbf{R}\left(\mathbf{X}^{\prime} \mathbf{X}\right)^{-1} \mathbf{X}^{\prime} \mathbf{Y}\right)
$$

and implies that $\mathrm{X}$ is non-singular. Unfortunately, there are a number of examples where this condition is not satisfied. Most notably, the case where one includes the full set of dummy 
variables in an equation and then the parameters are constrained to sum to zero (see Suits 1984).

Greene and Seaks (1991) show how the restricted least squares estimator may be computed even if $\mathrm{X}$ is singular by finding the direct solution of the first order conditions

$$
\left[\begin{array}{l}
\hat{\beta} \\
\lambda
\end{array}\right]=\left[\begin{array}{cc}
\mathbf{X}^{\prime} \mathbf{X} & \mathbf{R}^{\prime} \\
\mathbf{R}^{\prime} & 0
\end{array}\right]^{-1}\left[\begin{array}{c}
\mathbf{X}^{\prime} \mathbf{Y} \\
\mathrm{r}
\end{array}\right]
$$

where $\lambda$ is an $m \times 1$ vector of Lagrangean multipliers. Alternatively, the restricted least squares estimator can be found by using the reparameterization approach.

The reparameterization method also has the advantage of improving efficiency in computation. For example, in the linear regression case the LFUP estimation equation (8) requires the computation of two inverses: one of the matrix $\mathbf{Q}=\left(\mathbf{X}^{\prime} \tilde{\Sigma}^{-1} \mathbf{X}\right)^{-1}$ as well as for $\left(\mathbf{R Q R}^{\prime}\right)^{-\mathbf{1}}$. However, with the reparameterization the estimates require only one inversion $\left(\mathbf{A}^{\prime} \mathbf{X}^{\prime} \tilde{\Sigma}^{-1} \mathbf{X} \mathbf{A}\right)^{-1}$, see (7). Note that to compute the inverse of a matrix the number of individual computations is of the order of the cube of its dimension ${ }^{2}$.

An example of the gains from reparameterization can be found in Huang and Haidacher's (1983) paper in which they estimate a 13-equation demand system with 195 unrestricted parameters by applying a reparameterization of 92 restrictions defined on the unrestricted parameters. In this case the computation of the $\mathbf{Q}$ matrix would involve in the order of 7.4 million operations and computing (R Q $\left.\mathbf{R}^{\prime}\right)^{-1}$ approximately .8 million. Thus, these two inversions would require almost 8.2 million operations in total. However, $\left(\mathbf{A}^{\prime} \mathbf{X}^{\prime} \tilde{\Sigma}^{-1} \mathbf{X} \mathbf{A}\right)^{-1}$ is a (103 x 103) matrix and the number of operations needed for inversion is of the order of approximately 1.1 million. Thus, the reparameterized model estimate requires less than $1 / 8$ th of

\footnotetext{
2 At this point one might observe that the eigenvector computations needed for the conversions from LFUP to ROP and back do not warrant the reduction in dimensionality due to computational expense. The computation of the singular value decomposition requires the same order of computations that the inverse requires. Thus the conversion will be warranted on a purely computational efficiency if the method of computation requires more than one
} 
the computations associated with the traditional constrained optimization formula. In estimating demand systems with 30 or more commodities the differential in computations between the reparameterization method and the traditional technique levels off to $12 \%$. In addition, round off error may become a particularly difficult problem in the case of such large demand systems, due to the approximate multicollinearity in the X's which are defined as functions of prices, to the degree that it may not be possible to estimate the unrestricted model. ${ }^{3}$

\section{Example Applications}

This section illustrates the use of the conversion in restrictions from one type to the other. For convenience all these examples use linear regression models. In the first example, we demonstrate the use of the reparameterization method in the estimation of a system of demand equations subject to the exact linear restrictions defined by the symmetry and homogeneity of degree zero in the prices. The other two examples demonstrate how the ability to go from one form to the other can be used to estimate those models where there is a combination of restrictions that apply simultaneously.

\subsection{The reparameterization in the case of restricted demand equations}

Exact linear restrictions are used in the estimation of demand systems where economic theory implies a set of restrictions on the functional form of empirical demand equations.

Consider a system of two share equations as derived from a Translog cost function (Christensen and Greene 1976) which are subject to restrictions to insure cross price coefficient symmetry and where the cost function is homogeneous of degree one in prices

$$
\begin{gathered}
\mathbf{y}_{\mathbf{1}}=\alpha_{1}^{u}+\beta_{11}^{u} \mathbf{P}_{\mathbf{1}}+\beta_{12}^{u} \mathbf{P}_{\mathbf{2}}+\boldsymbol{\varepsilon}_{\mathbf{1}} \\
\mathbf{y}_{\mathbf{2}}=\alpha_{2}^{u}+\beta_{21}^{u} \mathbf{P}_{\mathbf{1}}+\beta_{22}^{u} \mathbf{P}_{\mathbf{2}}+\boldsymbol{\varepsilon}_{\mathbf{2}}
\end{gathered}
$$

Here, the $y^{\prime}$ s are the vectors of length $T$ of the cost shares and the P's are the $T$ by 1 vectors of

\footnotetext{
iteration.

${ }^{3}$ In the case of the symmetry restrictions alone Byron (1982) and Hirschberg (1992) demonstrate that the number of computations may even fall to a far smaller proportion if the estimated covariance structure is not required.
} 
the logs of the prices, $\beta_{j i}^{u}$ and $\alpha_{j}^{u}$ are the unrestricted parameters. The matrix form for the unrestricted model is

$$
\left(\begin{array}{l}
\mathbf{y}_{\mathbf{1}} \\
\mathbf{y}_{\mathbf{2}}
\end{array}\right)=\left(\begin{array}{rrrrrr}
\mathbf{l} & \mathbf{P}_{1} & \mathbf{P}_{2} & \mathbf{0} & \mathbf{0} & \mathbf{0} \\
\mathbf{0} & \mathbf{0} & \mathbf{0} & \mathbf{l} & \mathbf{P}_{\mathbf{1}} & \mathbf{P}_{2}
\end{array}\right)\left(\begin{array}{l}
\alpha_{1}^{u} \\
\beta_{11}^{u} \\
\beta_{12}^{u} \\
\alpha_{2}^{u} \\
\beta_{21}^{u} \\
\beta_{22}^{u}
\end{array}\right)+\left(\begin{array}{l}
\boldsymbol{\varepsilon}_{1} \\
\boldsymbol{\varepsilon}_{2}
\end{array}\right)
$$

where $\mathbf{t}$ is a column vector of length $T$ with all elements equal to 1 . Typically the errors to this model would be assumed to follow Zellner's (1962) seemingly unrelated regressions model.

The traditional approach to reparameterization of this model would be to apply restrictions of the form: the cross price restriction implies that $\beta_{12}=\beta_{21}$, and the homogeneity restriction implies that $\beta_{11}=-\beta_{12}, \beta_{21}=-\beta_{22}$, and $\alpha_{1}+\alpha_{2}=1$. If we incorporate the restrictions into our specification, we can then respecify (12) as

$$
\left(\begin{array}{l}
\mathbf{y}_{1} \\
\mathbf{y}_{2}
\end{array}\right)=\left(\begin{array}{rr}
\mathbf{l} & \left(\mathbf{P}_{1}-\mathbf{P}_{2}\right) \\
-\mathbf{l} & \left(\mathbf{P}_{1}-\mathbf{P}_{2}\right)
\end{array}\right)\left(\begin{array}{c}
\alpha_{1} \\
\beta_{11}
\end{array}\right)+\left(\begin{array}{l}
\mathbf{0} \\
\mathbf{l}
\end{array}\right)+\left(\begin{array}{l}
\boldsymbol{\varepsilon}_{1} \\
\boldsymbol{\varepsilon}_{2}
\end{array}\right)
$$

Obviously the parameters in (12) and (13) are not the same ones although it is common practice to refer to them by the same names. Here we suggest that the more appropriate notation when using a reparameterization would be to use a separate set of parameter definitions.

In this example, the reparameterization of the original problem was done solely for this specific case by manipulation of the equation. However, we can obtain an equivalent reparameterization using the automatic procedure discussed in Section 2. Define the restriction relationship $(\mathbf{R} \beta=\mathbf{r})$ in this case as 


$$
\left(\begin{array}{cccccc}
0 & 1 & 1 & 0 & 0 & 0 \\
0 & 0 & 0 & 0 & 1 & 1 \\
0 & 0 & 1 & 0 & -1 & 0 \\
1 & 0 & 0 & 1 & 0 & 0
\end{array}\right)\left(\begin{array}{c}
\alpha_{1} \\
\beta_{11} \\
\beta_{12} \\
\alpha_{2} \\
\beta_{21} \\
\beta_{22}
\end{array}\right)=\left(\begin{array}{l}
0 \\
0 \\
0 \\
1
\end{array}\right)
$$

The eigenvectors of $\mathbf{U}=\left(R^{\prime} R\right)$ are given as

$$
\mathrm{U}=\left(\begin{array}{rrrrrr}
0 & 0 & .71 & 0 & .71 & 0 \\
.27 & .5 & 0 & .65 & 0 & .5 \\
.65 & .5 & 0 & -.27 & 0 & -.5 \\
0 & 0 & .71 & 0 & -.71 & 0 \\
-.65 & .5 & 0 & .65 & 0 & -.5 \\
.27 & .5 & 0 & -.27 & 0 & 0
\end{array}\right)
$$

and the corresponding eigenvalues of $\mathbf{U}$ are given as

$$
\left(\begin{array}{llllll}
3.41 & 2 & 2 & .59 & 0 & 0
\end{array}\right)
$$

Thus, the last two columns of $\mathbf{U}$ make up $\mathbf{A}$, a matrix composed of the eigenvectors that correspond to the zero valued eigenvalues of $\mathbf{U}$ and each column can be multiplied by any scalar. Thus, we can rescale this matrix to be of the form

$$
\mathbf{A}=\left(\begin{array}{cc}
1 & 0 \\
0 & 1 \\
0 & -1 \\
-1 & 0 \\
0 & -1 \\
0 & 0
\end{array}\right)
$$

for $\mathbf{d}=\mathbf{R}^{+} \mathbf{r}$ we get

$$
\mathbf{d}=\left(\begin{array}{rrrr}
0 & 0 & 0 & .5 \\
.75 & -.25 & -.5 & 0 \\
.25 & .25 & .5 & 0 \\
0 & 0 & 0 & .5 \\
.25 & .25 & -.5 & 0 \\
.75 & .75 & .5 & 0
\end{array}\right)\left(\begin{array}{l}
0 \\
0 \\
0 \\
1
\end{array}\right)=\left(\begin{array}{l}
.5 \\
0 \\
0 \\
.5 \\
0 \\
0
\end{array}\right)
$$


$\mathbf{Z}=\mathbf{X A}$ results in

$$
\mathbf{Z}=\left(\begin{array}{cc}
-\mathbf{l} & \left(\mathbf{P}_{1}-\mathbf{P}_{2}\right) \\
-\mathbf{l} & \left(\mathbf{P}_{1}-\mathbf{P}_{2}\right)
\end{array}\right)
$$

and for $\mathbf{G}=\mathbf{Y}-\mathbf{X d}$

$$
\mathbf{G}=\left(\begin{array}{l}
\left(\mathbf{y}_{1}-.5 \mathbf{l}\right) \\
\left(\mathbf{y}_{2}-.5 \mathbf{l}\right)
\end{array}\right)
$$

It can be shown that the resulting estimates of $\beta$ will be the same from either reparameterization. The only difference is in the estimation of the $\alpha$ 's which will be set up differently in the second case, but which result in the same estimates of the restricted values of $\alpha$.

\subsection{The combination of both linear restrictions and reparameterizations.}

In this section we show that if combinations of linear restrictions and reparameterizations are imposed simultaneously then one can form a single equivalent set of linear restrictions or reparameterization. As demonstrated below, some applications lend themselves better to either reparameterization or to a linear restriction on the parameters.

If one has a LFUP and an ROP restriction on the same parameters it is possible to form a single set of LFUP restrictions by converting the ROP to a LFUP case and then the $\mathbf{R}_{\boldsymbol{i}}$ matrices are stacked on top of each other to form a new LFUP problem. If desired one can then convert the composite LFUP restriction to an ROP form. This is the case dealt with in 3.2.1 below. However, if we have an ROP case that is subject to an LFUP restriction in the reparameterized parameters $(\gamma)$ it is easiest to translate the LFUP on the new parameters to an ROP then multiply the $\mathbf{A}_{\boldsymbol{i}}$ matrices together to derive a single $\mathbf{A}$ matrix. This approach is illustrated below in 3.2.2. 


\subsubsection{The case of reparameterization and linear restrictions on the original model}

A frequently used reparameterization found in time series analysis involves the polynomial lag model as proposed by Almon (1965). In addition, it is often the case that incidental variables in a model are assumed to sum to zero. Here we demonstrate how these two types of restrictions can be included simultaneously.

In the case of the Almon polynomial lag model a series of lagged values of a variable are assumed to have parameters that are related to each other via a polynomial in the lags. A typical model of this type would be

$$
y_{t}=\beta_{0}+\beta_{1} X_{t}+\sum_{s=0}^{m} \beta_{2+s} Z_{t-s}+\varepsilon_{t}
$$

where the parameters $\beta_{2}$ to $\beta_{m}$ are subject to a reparameterization as given below if we assume a second order polynomial function:

$$
\beta_{2+s}=\phi_{0}+\phi_{1} s+\phi_{2} s^{2}
$$

Setting the length of the lag $m=5$ results in the following reparameterization equation $\left(\beta=\mathbf{A}_{1} \gamma+\mathbf{d}_{1}\right)$

$$
\left(\begin{array}{l}
\beta_{0} \\
\beta_{1} \\
\beta_{2} \\
\beta_{3} \\
\beta_{4} \\
\beta_{5} \\
\beta_{6}
\end{array}\right)=\left(\begin{array}{lllll}
1 & 0 & 0 & 0 & 0 \\
0 & 1 & 0 & 0 & 0 \\
0 & 0 & 1 & 0 & 0 \\
0 & 0 & 1 & 1 & 1 \\
0 & 0 & 1 & 2 & 4 \\
0 & 0 & 1 & 3 & 9 \\
0 & 0 & 1 & 4 & 16
\end{array}\right)\left(\begin{array}{l}
\gamma_{0} \\
\gamma_{1} \\
\gamma_{2} \\
\gamma_{3} \\
\gamma_{4}
\end{array}\right)+\left(\begin{array}{l}
0 \\
0 \\
0 \\
0 \\
0 \\
0 \\
0
\end{array}\right)
$$

where the new parameters $(\gamma)$ can be shown to be functions of the original parameters of the polynomial and the model

$$
\left(\begin{array}{lllll}
\gamma_{0} & \gamma_{1} & \gamma_{2} & \gamma_{3} & \gamma_{4}
\end{array}\right)^{\prime}=\left(\begin{array}{lllll}
\beta_{0} & \beta_{1} & \phi_{0} & \phi_{1} & \phi_{2}
\end{array}\right)^{\prime} .
$$

We can form the equivalent linear restriction as shown in Appendix B via the eigenvectors that correspond to the zero valued eigenvalues of $\mathbf{A}_{\mathbf{1}} \mathbf{A}_{\mathbf{1}}{ }^{\prime}$ a version of this is given by 


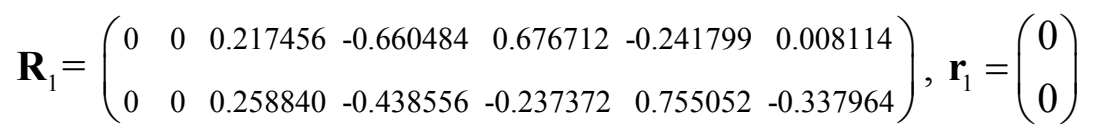

This implies that 2 restrictions are applied to all the parameters on the lagged values. An equivalent linear restriction matrix for the polynomial lag restriction is given in Fomby et al. (1984, pp. 376-377).

In addition let us assume that we also want to insure that the parameters for the lagged coefficients sum to zero. This may be the case when the Z's are dummy variables and we apply a restriction that dummy variables sum to zero (see Suits 1984). In this case, we would also want to apply the restriction that $\mathbf{R}_{2} \beta=\mathbf{r}_{2}$ or

$$
\left(\begin{array}{lllllll}
0 & 0 & 0 & 1 & 1 & 1 & 1
\end{array}\right)\left(\begin{array}{l}
\beta_{0} \\
\beta_{1} \\
\beta_{2} \\
\beta_{3} \\
\beta_{4} \\
\beta_{5} \\
\beta_{6}
\end{array}\right)=(0)
$$

Thus, we can add these constraints together to form a new $\mathbf{R}$ matrix by concatenating $\mathbf{R}_{1}$ and $\mathbf{R}_{2}$.

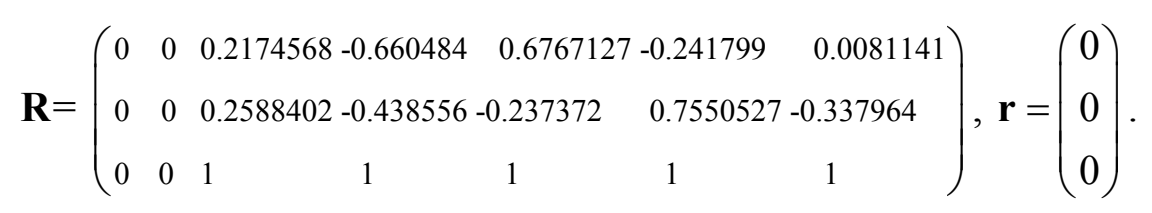

Thus the parameters are restricted by both constraints. Other typical additional parameter restrictions of the LFUP form in this case are those that ensure the polynomial ends and/or begins at zero (see for example Batten and Thornton 1983).

\subsubsection{The case when the reparameterizing equation is subject to linear restrictions}

This case concerns a proposal made by Poirier (1976, chapter 6) to use splines instead of simple polynomial lag functions. Suits et al (1978) demonstrate how spline functions can be estimated as constrained piecewise polynomials in parameters of a regression. Here the 
polynomial lag is a reparameterization of the original parameters and the assumption of a spline function involves placing restrictions on the new parameters from the reparameterization. Judge et al (1985, pp 364-365) propose that a sequence of two restricted estimations be used for this case. In the first, one estimates the parameters of the polynomial via the ROP form, then in the second stage, these parameters are made subject to a set of restrictions via the LFUP form of the reparameterized estimates. Here we demonstrate that the estimation of these models can be shown to be recast as a single ROP form that need only be estimated once.

To simplify our example we assume a bilinear spline for the lag coefficients from (21). The first step is to replace the polynomial lag with a set of two linear functions. We define the reparameterizing function as

$$
\begin{aligned}
& \beta_{2+s}=\gamma_{3} D_{1}+\gamma_{4} s D_{1}+\gamma_{5} D_{2}+\gamma_{6}(s-k) D_{2}, \\
& \text { where } D_{1}=1 \text {, when } s<k \text { and } D_{1}=0, \text { otherwise } \\
& \text { where } D_{2}=1 \text {, when } s \geq k \text { and } D_{2}=0, \text { otherwise }
\end{aligned}
$$

which implies that the lag function is two linear functions where the change over from one function to the other occurs at the knot defined by $k$. One starts at lag 0 and ends at lag $k-1$ and the other starts at lag $k$ and ends at lag $m$. This reparameterization would imply that when the knot was placed at $k=3, \beta=\mathbf{A}_{3} \gamma+\mathbf{d}_{3}$ is defined as

$$
\left(\begin{array}{l}
\beta_{0} \\
\beta_{1} \\
\beta_{2} \\
\beta_{3} \\
\beta_{4} \\
\beta_{5} \\
\beta_{6}
\end{array}\right)=\left(\begin{array}{llllll}
1 & 0 & 0 & 0 & 0 & 0 \\
0 & 1 & 0 & 0 & 0 & 0 \\
0 & 0 & 1 & 0 & 0 & 0 \\
0 & 0 & 1 & 1 & 0 & 0 \\
0 & 0 & 0 & 0 & 1 & 0 \\
0 & 0 & 0 & 0 & 1 & 1 \\
0 & 0 & 0 & 0 & 1 & 2
\end{array}\right)\left(\begin{array}{l}
\gamma_{0} \\
\gamma_{1} \\
\gamma_{2} \\
\gamma_{3} \\
\gamma_{4} \\
\gamma_{5}
\end{array}\right)+\left(\begin{array}{l}
0 \\
0 \\
0 \\
0 \\
0 \\
0 \\
0
\end{array}\right) .
$$

In spline functions one usually interrelates the separate functions by constraining the estimated functions to share common values at the knots. In the case of higher order polynomial functions such as a cubic, that the $1^{\text {st }}$ and $2^{\text {nd }}$ derivatives are equal at the knots as well. In this case we insure that the two linear functions meet at the knot by requiring that the relationship 
$\gamma_{2}+\gamma_{3} k=\gamma_{4}$ holds. In this case we then have a linear restriction on the reparameterized model coefficients defined as $\mathbf{R}_{\mathbf{4}} \gamma=\mathbf{r}_{\mathbf{4}}$ or:

$$
\left(\begin{array}{llllll}
0 & 0 & 1 & 3 & -1 & 0
\end{array}\right)\left(\begin{array}{l}
\gamma_{0} \\
\gamma_{1} \\
\gamma_{2} \\
\gamma_{3} \\
\gamma_{4} \\
\gamma_{5}
\end{array}\right)=(0)
$$

Additional restrictions to insure that the 1st and 2nd derivatives at the knots are equal would involve adding more rows to the $\mathbf{R}_{\mathbf{4}}$ matrix.

To accommodate this restriction on the reparameterized coefficients the linear restriction matrix $\left(\mathbf{R}_{4}\right)$ on these variables is transformed to the ROP form as $\gamma=\mathbf{A}_{4} \theta$ where $\mathbf{A}_{4}$ is the matrix made up of the eigenvectors corresponding to the zero valued eigenvalues of $\left(\mathbf{R}_{\mathbf{4}}{ }^{\prime} \mathbf{R}_{\mathbf{4}}\right)$ and $\theta$ are the free parameters that account for the lag function and the spline restrictions. The relationship between the original model parameters and these new parameters is $\beta=\mathbf{A}_{3} \mathbf{A}_{4} \theta$.

Note that the dimension of $\theta$ is 5 by 1 . A value for this new transformation matrix in this case is given as:

$$
\mathbf{A}_{3} \mathbf{A}_{4}=\left(\begin{array}{ccccc}
0 & 1 & 0 & 0 & 0 \\
1 & 0 & 0 & 0 & 0 \\
0 & 0 & 0 & .9533 & .0164 \\
0 & 0 & 0 & .6619 & .3277 \\
0 & 0 & 0 & .0790 & .9502 \\
0 & 0 & 1 & .0790 & .9502 \\
0 & 0 & 2 & .0790 & .9502
\end{array}\right)
$$

The estimation of the term structure of interest rates is a particular example of where this approach may be useful as often various combinations of restrictions are imposed simultaneously (see for example Steeley 1991 and Lin 2002). In estimating the term structure of 
interest rates the B-spline curve fitting technique is one of the most popular empirical methodologies and it is often the case that the spline curve is subject to additional linear restrictions. Another application is when the dependent variable is a limited dependent variable and the estimating relationship uses the method of splines (see for example Rosenberg et al. 2003).

\section{A test of linear restrictions using the reparameterized model}

An alternative method for testing linear hypothesis concerning regression parameters uses the ROP form of the model. From equation (A6) (in Appendix A) we have that the linear restriction $\mathbf{R} \beta=\mathbf{r}$ is equivalent to the solution of

$$
\beta=\mathbf{R}^{+} \mathbf{r}+\mathbf{A} \gamma
$$

where $\mathbf{R}^{+}$is the generalized inverse of $\mathbf{R}, \gamma$ is the vector of new parameters and $\mathbf{A}$ is the matrix of the eigenvectors corresponding to the zero valued eigenvalues of $\mathbf{R}^{\prime} \mathbf{R}$. We can make the substitution for $\beta$ in the regression equation $\mathbf{Y}=\mathbf{X}(\beta)+\varepsilon$ to obtain

$$
\mathbf{Y}=\mathbf{X}\left(\mathbf{R}^{+} \mathbf{r}+\mathbf{A} \gamma\right)+\varepsilon
$$

When we assume the restrictions hold we transform the independent variable from $\mathbf{Y}$ to $\mathbf{G}$ using $\mathbf{G}=\mathbf{Y}-\mathbf{X}\left(\mathbf{R}^{+} \mathbf{r}\right)$ and run a regression of the form

$$
\mathbf{G}=\mathbf{X}(\mathbf{A} \gamma)+\varepsilon
$$

However, in order to test the hypothesis that $\mathbf{R} \beta=\mathbf{r}$ we can define a stochastic parameter vector $\rho$ such that $\mathbf{R} \beta=\rho$. We can then estimate $\rho$ by using the following "augmented" reparameterized estimating equation which is equivalent to the unrestricted equation

$$
\mathbf{Y}=\mathbf{X}\left(\mathbf{R}^{+} \rho+\mathbf{A} \gamma\right)+\varepsilon
$$

and then form the Wald test for the null hypothesis that $\rho=\mathbf{r}$. This is equivalent to testing $\mathbf{R} \hat{\beta}=\mathbf{r}$ when $\hat{\beta}$ is the unrestricted estimate of $\beta$. When this hypothesis is true the reparameterized model is equivalent to the imposition of the linear equation, thus the assumption 
that $\rho=\mathbf{r}$. Consequently, we can test the restrictions directly by estimating $\hat{\rho}$ and test the null hypothesis that $\hat{\rho}=\mathbf{r}$ as a composite test or of the restrictions individually. If the original form of the restrictions are in the reparameterized form then we can construct the equivalent linear restrictions to find an $\mathbf{R}^{+}$matrix and vector $\mathbf{r}$ (where $\mathbf{r}=\mathbf{R d}$ ) and reformulate the model to test the restrictions.

\section{Conclusions}

This paper demonstrates that a reparameterized model can be found for any regression model subject to a set of linear restrictions and conversely that a linear restriction can be computed for any linear reparameterization of a regression model in a simple automatic fashion. Previous authors have not explicitly demonstrated this correspondence but only gave such relationships on a case by case basis.

The linear restricted model is the usual representation of the restricted regression model found in econometrics. However, reparameterization often helps in simplifying the estimation procedure which may be especially important when iterative methods are used for estimation as in the case of maximum likelihood, robust estimation and Bayesian methods. It would appear that textbook authors have tended to handle the reparameterization of restricted least squares problem in a somewhat hazy fashion because they lack a general method for the transformation of one form to the other. We hope that this is no longer the case.

This method of transformation may also be of use when considering non-linear restrictions. Although in this paper we have dealt exclusively with linear restrictions a common method for imposing non-linear restrictions is the linearization of the restrictions via a first order Taylor series approximation. Due to the automation of the conversion of these restrictions it would be a simple step to derive an equivalent new reparameterization at each iteration in the estimation and thus improve the computational characteristics in this case as well. 


\section{Appendix A. The Derivation of the ROP from the LFUP $(R \beta=r \rightarrow \beta=\mathbf{A} \gamma+\mathbf{d})$.}

We can solve the LFUP restriction equation, $\mathbf{R} \beta=\mathbf{r}$, using the general solution of a set of linear equations

$$
\beta=\mathbf{R}^{+} \mathbf{r}+\left(\mathbf{I}_{\mathbf{k}}-\mathbf{R}^{+} \mathbf{R}\right) \phi
$$

It can be shown that if a solution exists, this equation provides a solution (see Graybill 1983, Theorem 7.31), given some value for $\phi$ where $\mathbf{R}^{+}$is a generalized inverse of $\mathbf{R}$. If $\mathbf{R}$ is of rank equal to the number of rows $(m)$ then we can define $\mathbf{R}^{+}=\mathbf{R}^{\prime}\left(\mathbf{R} \mathbf{R}^{\prime}\right)^{-1}$. However, this solution does not furnish a reduction in dimensionality. $\left(\mathbf{I}_{\mathbf{k}}-\mathbf{R}^{+} \mathbf{R}\right)$ is of dimension $k \times k$.

To make this reduction we use the singular value decomposition (see chapter 4 of Lawson and Hanson 1974) of $\mathbf{R}$ whereby

$$
\mathbf{R}=\mathbf{H} \mathbf{C} \mathbf{U}^{\prime}
$$

where $\mathbf{H}$ is an orthogonal matrix with columns consisting of the eigenvectors of $\mathbf{R} \mathbf{R}^{\prime}, \mathbf{C}$ is an ( $m \times k)$ matrix of the square root of the eigenvalues of $\mathbf{R} \mathbf{R}^{\prime}$ on the first $m \times m$ diagonal, listed in decreasing order, $\mathbf{U}$ is an orthogonal $k \mathrm{x} k$ matrix with the columns consisting of the eigenvectors of $\mathbf{R}^{\prime} \mathbf{R}$. Thus, based on the information that rank of $\mathbf{R}$ is $m$ we have the following partition of $\mathbf{C}$ and $\mathbf{U}^{\prime}$

$$
\mathbf{R}=\mathbf{H}_{(\mathrm{m \times m})}\left(\mathbf{C}_{\mathbf{1}_{(\mathrm{m} \times \mathrm{m})}} \vdots \mathbf{0}_{(\mathrm{m \times j})}\right)_{(\mathrm{m \times k})}\left(\begin{array}{c}
\mathbf{U}_{\mathbf{1}_{(\mathrm{m} \times \mathrm{k})}} \\
\ldots \ldots \ldots \\
\mathbf{U}_{\mathbf{2}_{(\mathrm{j} \times \mathrm{k})}}
\end{array}\right)_{(\mathbf{k} \times \mathrm{k})}
$$

where $j=k-m$, and $m \times k \mathbf{U}_{\mathbf{1}}$ is made up from the $m$ rows of $\mathbf{U}^{\prime}$ associated with non-zero eigenvalues which are on the diagonal of $m \times m \mathbf{C}_{\mathbf{1}}$ and $\mathbf{U}_{\mathbf{2}}$ are the $j$ rows associated with the zero-valued eigenvalues.

Then as in Kennedy and Gentle (1980, pg 350) we rewrite our value for $\mathbf{R}^{+}$as

$$
\mathbf{R}^{+}=\mathbf{U} \mathbf{C}^{*} \mathbf{H}^{\prime}
$$

where $\mathbf{C}^{*}$ is defined as, 


$$
\mathbf{C}^{*}=\left(\begin{array}{c}
\mathbf{C}_{1}^{-1} \\
\cdots \\
\mathbf{0}
\end{array}\right),
$$

and

$$
\left(\mathbf{I}-\mathbf{R}^{+} \mathbf{R}\right)=\mathbf{U}_{2} \mathbf{U}_{2}^{\prime} .
$$

By substitution of (A5) into (A1) a value for $\beta$ is given as;

$$
\beta=\mathbf{R}^{+} \mathbf{r}+\mathbf{U}_{2} \mathbf{U}_{2}^{\prime} \phi
$$

where $\mathbf{U}_{2}{ }^{\prime} \phi$ is of dimension $j \times 1$. Thus, the equivalent new parameter set is

$$
\gamma=\mathbf{U}_{2}{ }^{\prime} \phi
$$

and use the following substitutions

$$
\mathbf{A}=\mathbf{U}_{2}
$$

This $\mathbf{A}$ is the submatrix of eigenvectors corresponding to zero valued eigenvalues of $\mathbf{R R}^{\prime}$ and

$$
\mathbf{d}=\mathbf{R}^{+} \mathbf{r}
$$

Appendix B The derivation of the LFUP from the ROP $(\beta=\mathbf{A} \gamma+\mathbf{d} \rightarrow \mathrm{R} \beta=\mathbf{r})$.

Following Fomby, Hill and Johnson (1984, pages 85 and 393) premultiply both sides of the equation

$$
\beta=\mathbf{A} \gamma+\mathbf{d}
$$

by the Moore-Penrose generalized inverse of A;

$$
\mathbf{A}^{+} \beta=\gamma+\mathbf{A}^{+} \mathbf{d}
$$

where when $\mathbf{A}$ is of full column $\operatorname{rank}\left(\operatorname{rank}\right.$ of $\mathbf{A}$ is $j$ ) $\mathbf{A}^{+}$can be defined by

$$
\mathbf{A}^{+}=\left(\mathbf{A}^{\prime} \mathbf{A}\right)^{-1} \mathbf{A}^{\prime}
$$


Thus, we can solve for $\gamma$ as a function of $\beta$

$$
\gamma=\mathbf{A}^{+}(\beta-\mathbf{d})
$$

by substitution of (B4) in (B1), we get a linear equation in only $\beta$

$$
\left(\mathbf{I}-\mathbf{A} \mathbf{A}^{+}\right) \beta=\left(\mathbf{I}-\mathbf{A} \mathbf{A}^{+}\right) \mathbf{d}
$$

However, $\left(\mathrm{I}-\mathbf{A} \mathbf{A}^{+}\right)$is of dimension $k$ by $k$, but it is not of full row rank and it is unsatisfactory for use in the traditional restricted least squares solution, thus it is necessary to reduce the dimensionality. To make this reduction we follow the proof in Appendix A and use the singular value decomposition of $\mathbf{A}$ whereby we can find a triplet of matrices of the form

$$
\mathbf{A}=\mathbf{H} \mathbf{C} \mathbf{U}^{\prime}
$$

where in this case $\mathbf{H}$ is a $k \mathbf{x} k$ orthogonal matrix with columns consisting of the eigenvectors of $\mathbf{A A}^{\prime}, \mathbf{C}$ is an $(k \times j)$ matrix with the square root of the eigenvalues of $\mathbf{A}^{\prime} \mathbf{A}$ on the top $j \mathrm{x} j$ diagonal, $\mathbf{U}$ is an orthogonal $j \mathrm{x} j$ matrix with the columns consisting of the eigenvectors of $\mathbf{A}^{\prime} \mathbf{A}$. Thus, based on the information that rank of $\mathbf{A}$ is $j$ we have the following partition of $\mathbf{C}$ and $\mathbf{U}^{\prime}$

$$
\mathbf{A}=\left(\begin{array}{lll}
\mathbf{H}_{1_{(\mathrm{k} \times \mathrm{j})}} & \vdots & \mathbf{H}_{2_{(\mathrm{k} \times \mathrm{m})}}
\end{array}\right)_{(\mathrm{kxk})}\left(\begin{array}{c}
\mathbf{C}_{1_{(\mathrm{kxj})}} \\
\cdots \cdots \\
\mathbf{0}_{(\mathrm{m} \times \mathrm{j})}
\end{array}\right)_{(\mathrm{k} \times \mathrm{j})}(\mathbf{U})_{(\mathrm{j} \times \mathrm{j})}
$$

where $\mathbf{H}_{\mathbf{1}}$ are the $j$ columns of $\mathbf{H}$ associated with non-zero eigenvalues which are on the diagonal of $\mathbf{C}_{1}$ and $\mathbf{H}_{2}$ are the $k$-j rows associated with the zero-valued eigenvalues.

Following Kennedy and Gentle (1980, pg 350) we rewrite for $\mathbf{A}^{+}$as

$$
\mathbf{A}^{+}=\mathbf{U} \mathbf{C}^{*} \mathbf{H}^{\prime}
$$

where $\mathbf{C}^{*}$ is defined as,

$$
\mathbf{C}^{*}=\left(\begin{array}{lll}
\mathbf{C}_{1}^{-1} & \vdots & \mathbf{0}
\end{array}\right)
$$

and 


$$
\left(\mathbf{I}-\mathbf{A A}^{+}\right)=\mathbf{H}_{2} \mathbf{H}_{2}{ }^{\prime} .
$$

By substitution of (B9) in (B5) the restriction in terms of $\beta$ is given as;

$$
\mathbf{H}_{2} \mathbf{H}_{2}{ }^{\prime} \beta=\mathbf{H}_{2} \mathbf{H}_{2}{ }^{\prime} \mathbf{d}
$$

and by premultiplying both sides by $\mathbf{H}_{\mathbf{2}}{ }^{\prime}$ we get

$$
\mathbf{H}_{2}{ }^{\prime} \beta=\mathbf{H}_{2}{ }^{\prime} \mathbf{d}
$$

Thus $\mathbf{R}=\mathbf{H}_{\mathbf{2}}{ }^{\prime}$ and $\mathbf{r}=\mathbf{H}_{\mathbf{2}}{ }^{\prime} \mathbf{d}$ in the equation given as $\mathbf{R} \beta=\mathbf{r}$ form and we recall that $\mathbf{H}_{\mathbf{2}}{ }^{\prime}$ is the transpose of the matrix of eigenvectors of $\mathbf{A A}^{\prime}$ that correspond to the zero valued eigenvalues of $\mathbf{A A}^{\prime}$ matrix. 


\section{References}

Almon, S. (1965), "The Distributed Lag Between Capital Appropriations and Expenditures", Econometrica, 33, 178-196.

Batten, D.S. and D. Thornton (1983), "Polynomial Distributed Lags and the Estimation of the St Louis Equation”, Federal Bank of St Louis Review, April, 13-25..

Byron, R. P. (1982), "A note on the estimation of symmetric systems", Econometrica, 50, 1573 1575.

Christensen, L. R. and W. H. Greene (1976), "Economies of Scale in U.S. Electric Power Generation", Journal of Political Economy, 84, 655-676.

Davidson, R. and J. G. MacKinnon (1993), Estimation and Inference in Econometrics, Oxford University Press, New York, NY.

Fomby, T. B., R. C. Hill, S. R. Johnson (1984), Advanced Econometric Methods, SpringerVerlag, New York, NY.

Graybill, F. A. (1983), Matrices with Applications in Statistics, 2nd Edition, Wadsworth, Belmont, CA.

Greene, W. H. (2003), Econometric Analysis, 5th Edition, Macmillan, New York.

Greene, W. H. and T. G. Seaks (1991), "The restricted Least Squares Estimator A pedagogical Note", Review of Economics and Statistics, 563-567.

Griffiths, W. E., C. O'Donnell and A. Tan Cruz (2000), "Imposing regularity conditions on a system of cost and factor share equations", The Australian Journal of Agricultural and Resource Economics, 44, 107-127.

Griffiths, W. E., R. C. Hill and G. G. Judge (1993), Learning and Practicing Econometrics, John Wiley \& Sons, New York, NY.

Hirschberg, J. G. (1992), "A computationally efficient method for bootstrapping systems of demand equations a comparison to traditional techniques", Statistics and Computing, 2, $19-24$.

Huang, K. S. and R. C. Haidacher (1983), "Estimation of a Composite Food Demand System for the United States", Journal of Business \& Statistics, 1 285-291.

Johnston, J. and J. DiNardo (1997), Econometric Methods, 4th Edition, McGraw-Hill, New York, NY.

Judge, G. G., W. E. Griffiths, R. C. Hill, H. Lükepohl, T.-C. Lee (1985), The Theory and Practice of Econometrics, 2nd Edition, John Wiley and Sons, New York, NY.

Kennedy W.J. and J. E. Gentle (1980), Statistical Computing, M. Dekker, New York. 
Lawson, C.L. and R.J. Hanson (1974), Solving Least Squares Problems, Prentice-Hall, Englewood Cliffs, NJ.

Lin, Bing-Hui (2002), "Fitting term structure of interest rates using B-splines: the case of Taiwanese Government bonds", Applied Financial Economics, 12, 57-75.

Mantell, E. H. (1973), "Exact Linear Restrictions on Parameters in the Classical Linear Regression Model", The American Statistician, 27, 86-87.

Poirier, D. J., (1976), The Econometrics of Structural Change, North-Holland, Amsterdam, The Netherlands.

Rosenberg P. S., H. Katki, C. Swanson, L. Brown, S. Wacholder and R. Hoover, (2003), "Quantifying epidemiologic risk factors using non-parametric regression: model selection remains the greatest challenge, Statistics in Medicine, 22, 3369-3381.

Ruud, P. A., (2000), An Introduction to Classical Econometric Theory, Oxford University Press.

Steeley, J. (1991), "Estimating the Gilt-edged Term Structure: Basic Splines and Confidence Intervals", Journal of Business Finance and Accounting, 18, 513-529.

Suits, D. B. (1984), "Dummy Variables: Mechanics V. Interpretation”, The Review of Economics and Statistics, 66, 177-180.

Suits, D. B.; A. Mason, L. Chan (1978), "Spline Functions Fitted by Standard Regression Methods", The Review of Economics and Statistics; 60, 132-39.

Tintner, G. (1952), Econometrics, John Wiley and Sons, New York, NY.

Zellner, A. (1962), “An Efficient Method of Estimating Seemingly Unrelated Regressions and Tests for Aggregation Bias.”, Journal of the American Statistical Association, 57, 348368. 